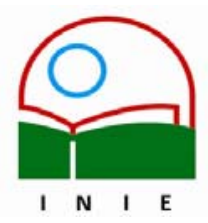

Universidad de Costa Rica

Facultad de Educación

Instituto de Investigación en Educación

ACTUALIDADES INVESTIGATIVAS EN EDUCACION

\title{
LA VALIDACIÓN EXTERNA Y EL ROL QUE DESEMPEÑA EL EQUIPO DE PARES EXTERNOS EN LOS PROCESOS DE AUTOEVALUACIÓN DE CARRERAS
}

\begin{abstract}
Carlos Mora Aguilar ${ }^{1}$
José Miguel Gutiérrez Mata²

Resumen: Este artículo ofrece una serie de consideraciones por seguir, en la fase final de los procesos de autoevaluación, denominada validación externa o evaluación externa. Por consiguiente, esta fase del proceso es guiada entre otras personas, por un equipo de evaluadores conformado por personal académico y profesional de alto nivel en su disciplina, que participa en la validación externa de carreras.

Centrados en el equipo de evaluación externa, el artículo presenta una serie de características y funciones con que éste debe contar, con el propósito de ofrecer un insumo general para todas aquellas personas, que dentro y fuera del país, tengan la intención de conformar un equipo de evaluación externa, como también a todas aquellas que tengan la inquietud por conocer sobre estos temas.
\end{abstract}

Palabras clave: AUTOEVALUACIÓN/ CARRERAS/ EQUIPO DE PARES EXTERNOS/VALIDACIÓN EXTERNA/ PARES EXTERNOS/ PAR EXTERNO/

Abstract: This article offers a series of considerations to follow, in the final phase of the autoevaluation processes, denominated external validation or external evaluation. Therefore, this phase of the process is guided, among other people, conformed by a high-level crew of academic and professional evaluators in their discipline.

Centered around the team of external evaluation, the article presents a series of characteristics and functions which it must count on, in order to offer general rudiments for all of those who, in and outside the country, may have interest to know about these issues.

Key words: AUTOEVALUATION/ CAREERS, EXTERNAL PAIRS CREW/ EXTERNAL VALIDATION/ EXTERNAL PAIRS/ EXTERNAL PEER/

\footnotetext{
${ }^{1}$ Magíster en Administración de Negocios por la Universidad Interamericana de Costa Rica. Licenciado y Bachiller en Ciencias Políticas por la Universidad de Costa Rica. Actualmente se desempeña como Docente-Investigador del Centro de Evaluación Académica de la Universidad de Costa Rica, en la labor de asesoría curricular y en procesos de autoevaluación con fines de mejora y con fines de acreditación. También realiza capacitación de pares externos a nivel institucional. Cuenta con el título de Evaluador Externo Internacional, otorgado por la Unión de Universidades de América Latina y los Comités Interinstitucionales para la Evaluación de la Educación Superior, México 2003. Brinda asesoría Correo electrónico: cemora@cariari.ucr.ac.cr

${ }^{2}$ Magister en Evaluación Educativa, Licenciado en Historia y Bachiller en la Enseñanza de los Estudios Sociales, además cuenta con el título de Evaluador Externo Internacional, otorgado por la Unión de Universidades de América Latina y los Comités Interinstitucionales para la Evaluación de la Educación Superior, México 2003. Actualmente se desempeña como docente-investigador en el Centro de Evaluación Académica de la Universidad de Costa Rica, en la labor de asesoría curricular y en procesos de autoevaluación con fines de mejora y con fines de acreditación. También realiza capacitación de pares externos a nivel institucional. Correo electrónico: imgutier@cariari.ucr.ac.cr
}

Artículo recibido: 14 de julio, 2005

Aprobado: 19 de setiembre, 2005 


\section{Presentación}

El documento que a continuación se presenta, tiene el objetivo de brindar información que oriente a las personas interesadas, a formar parte de equipos de evaluación externa y, en general, a las unidades académicas, respecto a la evaluación externa. Esta se constituye en la fase final del proceso de autoevaluación, al que voluntariamente se someten algunas unidades académicas en la Universidad de Costa Rica.

Es oportuno indicar que el proceso de autoevaluación y, con ello, la validación externa, se circunscribe a una carrera, a un programa o a una institución educativa. Para tal efecto, este documento hará referencia, solo a los procesos de autoevaluación de carreras.

Este trabajo reúne las experiencias vividas por los autores, durante los últimos años, en materia de evaluación externa, tanto en los ámbitos internacional como nacional. En el ámbito internacional, se retoma la experiencia de los autores en los Talleres Latinoamericanos de Capacitación de Evaluadores Externos organizado por la Unión de Universidades Latinoamericanas (UDUAL), en la Universidad Autónoma del Estado de Morelos y en la Universidad de Guanajuato, en el año 2002. El modelo aplicado en estas universidades corresponde al Análisis Estructural Integrativo de Organizaciones Universitarias (AEIOU) (González, 2000), para evaluación externa, así como la participación en el I Taller de Coordinadores de Evaluación Externa de la Educación Superior de la Escuela Politécnica Nacional, Quito Ecuador.

En el ámbito nacional, se realizó la evaluación externa, en el año 2003, del programa de Maestría Académica en Geología con énfasis en Manejo de Recursos Hídricos e Hidrogeología de la Escuela Centroamericana de Geología. A esta experiencia, se suman las asesorías de los autores, realizadas como parte del Centro de Evaluación Académica de la Universidad de Costa Rica, en materia de autoevaluación, autorregulación o mejoramiento de carreras, acreditación y la visita de pares externos.

Los anteriores insumos, junto con revisiones bibliográficas referentes a la evaluación externa, contenidas en este artículo, constituyen un recurso valioso para las unidades académicas que reciben la visita del equipo de pares externos y para las personas que eventualmente, podrían desempeñarse como tales, ya sea dentro o fuera del territorio nacional.

En la actualidad, en la Universidad de Costa Rica, 26 carreras se encuentran en procesos de autoevaluación, seis carreras acreditadas con el Sistema Nacional de Acreditación de la Educación Superior (SINAES) y tres carreras del área de la Ingeniería, 
acreditadas de forma equivalente por la Canadian Engineering Acreditation Board (CEAB) y cuenta con una carrera que se autoevalúo con fines de mejoramiento por medio del Sistema Centroamericano de la Evaluación de la Educación Superior (SICEVAES). Finalmente, los aspectos que en este documento se consignan, tienen un carácter general, que al ser conocidos por el personal académico, pueden ser útiles para su formación en estos procesos.

\section{El proceso de autoevaluación y la acreditación en la Universidad de Costa Rica}

El desarrollo y aseguramiento de la calidad en las instituciones de Educación Superior, son asuntos fundamentales para la realización de cambios, con fines de mejorar, tanto a lo interno como a lo externo de ellas. En la Universidad de Costa Rica, el aseguramiento sistemático de la calidad, se enriquece a partir de la última década del siglo XX, desde su integración en la conformación del Sistema Nacional de Acreditación en 1995, y la motivación dada a la comunidad universitaria para participar en los procesos de autoevaluación-autorregulación para el mejoramiento y la acreditación por parte de la Rectoría, Vicerrectoría de Docencia y, por ende, el Centro de Evaluación Académica (Vicerrectoría de Docencia, 2001).

Además, el Consejo Universitario consideró “... establecer mecanismos de evaluación externa para carreras y programas universitarios que puedan dar fe pública de la calidad académica que los caracteriza (acreditación)", así como "que las universidades estatales cuenten en su interior con sistemas formales adecuados de autoevaluación integral y que entren en un proceso permanente de examen y análisis de cumplimento de objetivos intermedios y finales de carreras y programas..." (Vicerrectoría de Docencia, 2001, p. 1).

De lo anterior se desprenden dos conceptos primordiales. El primero se refiere al proceso de autoevaluación, el cual es concebido en la Universidad de Costa Rica, como

un proceso voluntario de reflexión y análisis participativo por medio del cual una unidad académica realiza un análisis integral de su quehacer, de acuerdo con criterios de calidad, previamente establecidos. Este proceso es conducido por los propios integrantes de la unidad, en el marco de los fines, principios y funciones de la Universidad, la misión de la unidad académica y el papel protagónico de la institución. (CEA, 1999, p. xiii)

El proceso de autoevaluación no debe dejar de lado, en su componente conceptual y operativo, la importancia de varios elementos, entre los que destacan como pilares de éxito 
la reflexión crítica, la participación y fundamentalmente la transparencia, acerca del quehacer académico y de la gestión, en referencia con los objetivos previamente establecidos.

Aunado a lo anterior, el proceso de autoevaluación que realiza una carrera, se encuentra incompleto, hasta tanto no sea validado por un equipo conformado por pares evaluadores externos, en la fase denominada evaluación externa. Esta constituye una fase posterior al proceso de autoevaluación y se ejecuta por parte de un equipo de personas frecuentemente denominadas "pares externos" o evaluadores externos. En este documento preferimos utilizar la denominación de equipo de evaluación externa, por considerarla más precisa, en cuanto a la función por realizar.

La evaluación externa es definida como el análisis crítico y valorativo del proceso de autoevaluación de una carrera, por parte del equipo de pares externos a la instancia en evaluación y se basa esencialmente en tres tipos de evidencias: el informe integral de la autoevaluación, la información adicional recopilada en el proceso y las observaciones in situ realizadas durante la visita a la instancia que se autoevaluó. En otras palabras, la evaluación externa y, por ende, la validación del proceso de autoevaluación, desarrollada por el equipo de pares, involucra las fases de recopilación de datos, análisis, interpretación y juicio de esa información, y finalmente, de elaboración de un informe, donde se concretan las valoraciones de fortalezas y debilidades, con el fin de aportar propuestas de mejoramiento.

La evaluación externa tiene como propósito disponer de una validación externa del proceso de autoevaluación, para contribuir con el mejoramiento continuo de la calidad de una carrera (Kells, 1997). En general, los propósitos que cumple la evaluación externa son:

1. Valorar el proceso de autoevaluación, en relación con el diseño metodológico y su ejecución, los resultados y las acciones de mejoramiento.

2. Prevenir el sesgo subjetivo, cuando los implicados en la autoevaluación caen en la autocomplacencia.

3. Retroalimentar el proceso de autorregulación, por medio del aporte de recomendaciones que fortalezcan el plan de mejoramiento trazado.

4. Recomendar si se debe otorgar o no la acreditación, en el caso de que la autoevaluación atienda además al propósito externo de la acreditación.

5. Enriquecer la visión interna de la carrera, aportando una perspectiva experta diferente. Esta visión también puede aportar una imagen más integral de la carrera evaluada. 
En este sentido, la evaluación externa proporciona información sobre la objetividad, validez, credibilidad y confiabilidad de los procedimientos y resultados de la autoevaluación, tanto ante agentes externos, como ante la propia carrera. Asimismo, es posible identificar otros aspectos importantes por evaluar que no habían sido considerados inicialmente y también obtener sugerencias respecto a las estrategias de cambio propuestas. (UDUAL. 1er Taller, 2000)

\section{El equipo de evaluación externa}

El proceso de evaluación externa se realiza en equipo, ya que de esta forma se obtiene más rigor en el contraste de la información, más fuerza social en la tarea y más apoyo psicológico y resistencia a las presiones que podrían presentarse en la visita dentro de la carrera autoevaluada.

El equipo de evaluación externa está conformado por personas académicas y profesionales de alto nivel en su disciplina o en la gestión universitaria, relacionadas por su área disciplinar y afines a la carrera que se ha autoevaluado. Se nombran de manera ad hoc para participar en la validación externa de la autoevaluación de carreras y debe estar conformado de tal manera que les sea posible emitir un juicio valorativo, sobre la calidad de la carrera determinada por el proceso de autoevaluación, lo cual implica la capacidad del equipo para analizar los distintos aspectos que deben involucrarse.

Finalmente, la persona que integra un equipo de evaluación externa, responde a un perfil ya determinado, que de acuerdo con Moisés Silva (1999), tendría que contar con:

- reconocido alto nivel o liderazgo en su campo.

- experiencia/conocimiento en la academia, la administración, la evaluación institucional y el ejercicio de la profesión.

- capacidad para comunicarse y leer el idioma del país.

- intachable reputación ética/profesional.

Este equipo se constituye en una comisión de trabajo, en la cual una de las personas integrantes se desempeña como coordinadora. Dicha persona estará encargada de orientar el trabajo, de acordar con las autoridades de la institución la fecha de la visita, de velar para que ésta se realice en conformidad con los criterios y procedimientos establecidos por la institución o por el ente acreditador y de elaborar, junto con quienes integren el equipo 
evaluador, un informe escrito. Este equipo evaluador se caracteriza por tener independencia (no poseer vínculos contractuales como formales o equivalentes ni compromisos en el campo académico), representatividad de especialidades (disciplinas, experiencias profesionales, gestión-administración académica, evaluación y, según el caso, competencias en otros campos específicos).

Los siguientes aspectos deben considerarse, como una base común que debe tener el equipo de evaluación externa, en todo momento:

1. Respeto a la institución y su personal: Los pares deben respetar la institución y la carrera que evalúan para que mejoren. Deben respetar su misión, sus objetivos, sus características, su cultura. Una evaluación no tiene como objetivo uniformizar las instituciones o las carreras, tampoco se debe comparar la institución que evalúan con otras de la misma índole y particularmente con su propia institución.

2. Deseo de comprender antes de juzgar: Los evaluadores externos deben esforzarse en comprender bien la situación de la institución que evalúan con el fin de identificar correctamente sus fortalezas y debilidades. Eso se logra al leer cuidadosamente el informe de autoevaluación y durante la visita, al consultar los documentos disponibles y hacer las preguntas necesarias para esclarecer los puntos oscuros.

3. Rigor en el trabajo. Para evaluar es necesario tener puntos de referencia. Usualmente esos puntos toman la forma de criterios, normas y estándares que constituyen un conjunto de características que se encuentran en instituciones o programas de calidad. El trabajo de validación que realizan los pares debe ser riguroso, con respecto a la situación de una institución o programa y con los referentes de calidad con que se evalúa.

4. Discreción y distancia. Para que una evaluación sea eficaz y creíble, es importante que la comunicación entre la institución universitaria y los pares sea franca y abierta. Eso requiere que los pares se comprometan a guardar confidencialmente las informaciones que reciben. Aunque la visita debe desarrollarse en un clima cordial y transparente, es importante que los pares mantengan su distancia respecto a la institución, sino su trabajo perderá su credibilidad. 
5. Esfuerzo por liberarse de prejuicios y sesgos. Los pares deben hacer todo el esfuerzo posible para ser "objetivos en sus evaluaciones y juicios. Eso parece obvio, pero requiere un esfuerzo y cuidado particular.

\section{La visita del equipo de evaluación externa}

La visita del equipo de evaluación externa es un momento muy importante dentro de la autoevaluación. En un tiempo limitado, las personas evaluadoras comprobarán la exactitud de la documentación enviada por la carrera y adjuntarán información complementaria que consideren pertinente en su informe escrito. Las fuentes de información más relevantes para la evaluación externa son: el informe de autoevaluación, el archivo físico y digital preparado por la Comisión de Autoevaluación, las instalaciones de la institución, la población estudiantil de la carrera, el personal docente (que facilita, asesora y da tutoría académica), el personal directivo y la población administrativa, las personas graduadas, el sector empleador y otras personas que sean requeridas en el proceso. Para tal efecto, durante la visita, la unidad académica pondrá a disposición del equipo de evaluación, la información que sea requerida, los contactos necesarios con personas o instancias ajenas a la carrera, pero que se relacionan con ella y les brindará adecuado apoyo logístico.

La visita a las instalaciones de la institución tiene una duración aproximada de dos a cuatro días, dependiendo de los objetivos de la autoevaluación, su focalización y amplitud de los estudios. Realizar esta visita implica una serie de labores fundamentales, tales como:

1. Reuniones privadas de coordinación, entre el equipo de evaluadores externos y la comisión de autoevaluación, cuando sea necesario.

2. Reuniones con autoridades que tengan a su cargo diferentes instancias, tales como Rectoría, vicerrectoría, decanatura y oficina administrativa, entre otras.

3. Entrevistas con distintas personas de la unidad académica, tales como la directora o director (que también puede ser la decana o el decano, según el caso), el personal administrativo, el personal docente, el estudiantado, las personas graduadas y otros grupos de interés.

4. Visitas a las instalaciones (aulas, laboratorios, talleres, bibliotecas, sodas y otros).

5. Revisión de documentos (plan de estudio, programas de curso, tesis de grado, estudios sobre la carrera, proyectos y publicaciones, normativa, muestra de pruebas escritas, encuestas, entre otros). 
6. Presentación de un informe oral a las autoridades y a la Comisión de Autoevaluación en el término de la visita, el cual ofrece carácter preliminar y tiene como fin brindar un balance entre lo estipulado en el informe del proceso de autoevaluación y la visita realizada.

Antes de iniciar la visita, el equipo de evaluación externa, debe reunirse y planificar las estrategias por realizar durante la visita y, además, elegir a quién coordinará el equipo. Además debe realizar un programa de visita, el cual es elaborado por la institución en coordinación con el equipo mismo, siendo los requerimientos de esta última los que, en principio, deben prevalecer. Además no podrá divulgar información relacionada con la institución y el programa objeto de examen.

Es importante indicar que, como requisito previo a la realización de la visita del equipo de evaluación externa, la Comisión de Autoevaluación de la unidad académica debe disponer de:

1. Información amplia y adecuada para la comunidad de la unidad académica sobre el proceso realizado y los objetivos de la visita y el programa establecido.

2. Plena disponibilidad de autoridades y jefaturas universitarias.

3. Disposición para la consulta sobre el proceso de las diferentes poblaciones de la unidad académica. Entre ellas, población docente, estudiantil, administrativa, graduada, especialmente aquella pertinente al proceso efectuado.

4. Una funcionalidad plena y normal de la institución y por ende de la unidad académica, con particular atención al área en que se ha focalizado el proceso.

\section{El informe de validación por parte del equipo de evaluación externa}

Como uno de los puntos finales de la visita del equipo de evaluadores externos, se encuentra la elaboración del informe que, generalmente, consta de dos momentos.

En un primer momento, se hace un informe preliminar que se expone oralmente ante las autoridades institucionales y las personas representantes de la unidad académica. En él se hace un recuento de las opiniones sobre lo presentado en el informe de autoevaluación y lo constatado durante la visita de campo, respetando el contexto de los criterios establecidos para el proceso de autoevaluación. 
En un segundo momento, y con base en los criterios anteriores, se realiza el informe escrito, que tiene como fin emitir un juicio valorativo de la calidad de la carrera autoevaluada. El informe final escrito debe contener las siguientes características (Peralta, s.f.):

- $\quad$ Ser conciso, analítico y valorativo en relación con los propósitos definidos para la autoevaluación y los resultados obtenidos.

- $\quad$ Ser claro y preciso con respecto a la valoración de la calidad de la carrera.

- Informar sobre el cumplimiento del programa de visita del equipo de evaluación externa, sus logros y limitaciones.

- Valorar la gestión misma del proceso de autoevaluación, sus resultados y las acciones para el mejoramiento.

- Emitir una recomendación acerca del cumplimiento de los propósitos establecidos para el proceso de autoevaluación realizado.

- $\quad$ Finalmente, el informe oral y el informe escrito deben ser coherentes y éste último debe retomar y ampliar los aspectos anotados en el informe oral.

El informe escrito debe realizarse de manera inmediata a la visita de campo por parte del equipo de evaluación externa, bajo la conducción de quien coordina el equipo. Este informe se puede entregar posterior a la visita, en un lapso no mayor de un mes, para lo cual deben tomarse en cuenta algunas de las siguientes sugerencias:

1. Evitar la redacción meramente descriptiva; describir sólo para fundamentar las valoraciones y juicios, sobre todo, cuando resulten recomendaciones.

2. Fundamentar los juicios con respecto al daño que causa a la institución un problema advertido, y a los beneficios que le traerá la recomendación correspondiente.

3. Al referirse a un proceso o una estructura organizativa, se deberán contar con recomendaciones y sugerencias para su aplicación.

4. Al referirse a un proceso o una estructura organizativa, se deberán precisar los órganos involucrados y la congruencia existente con la normatividad establecida.

5. Al mencionar una dependencia o unidad orgánica, anotar su nombre exacto, su línea de autoridad y subordinación. 
6. Si la dependencia aludida no aparece en el organigrama, deberá explicarse; si es de reciente creación, se debe precisar la fecha; si cambió de nombre, funciones o nivel, es necesario señalar la situación anterior y la nueva.

7. Conviene una redacción explícita y evaluativa y una mayor atención a lo que se quiere decir comunicar.

Finalmente, el informe escrito es la esencia de la experiencia vivida en la visita y la lectura profunda del informe facilitado. Compila tanto, los insumos de la autoevaluación de la carrera como el juicio valorativo del equipo de pares externos, con el fin de obtener una visión integral del proceso realizado.

\section{Conclusiones}

Todo proceso de autoevaluación se encuentra incompleto, hasta que es validado por el equipo conformado por pares evaluadores externos, en la fase denominada evaluación externa, la cual es posterior al proceso de autoevaluación.

Esta validación toma como referencia el contenido del informe de autoevaluación, lo observado en la visita y, a partir de ello, emite juicios respecto al cumplimiento de los objetivos declarados, de los referentes aceptados y hace sugerencias sobre las recomendaciones o directrices de mejoramiento.

Dadas la responsabilidad y seriedad académicas que amerita la validación del equipo de evaluación externa, se debe considerar para estos procesos, una serie de características por cumplir como lo son: respeto a la institución y su personal, deseo de comprender antes de juzgar, rigor en el trabajo, discreción y distancia y esfuerzo por liberarse de prejuicios y sesgos.

El papel que representa una persona como integrante de un equipo de pares externos, conlleva una gran responsabilidad implícita, debido al grado de compromiso y suspicacia que debe tener para verificar y contrastar lo que posee, como insumo (informe), visita "in situ" (campo de acción) y el juicio valorativo que debe emitir al final.

La experiencia obtenida en la Universidad de Costa Rica en la formación de personas que integran un equipo de pares externos, permite a los autores, definir que para ejercer un rol de par externo, es necesario contar con conocimientos en el área disciplinar de la carrera, 
carácter investigativo, capacidad de síntesis y de trabajo en equipo, y además, proyectar el liderazgo y la confianza que les permitan garantizar un trabajo serio.

El ideal por alcanzar en estos procesos, será el de encontrar la coherencia entre el informe escrito de autoevaluación y lo encontrado en la visita "in situ", logrando reflejar, en todo momento, que el proceso de autoevaluación y validación externa realizada, refleje valores éticos, transparencia en la información, comunicación abierta entre las poblaciones involucradas y profesionalismo en el trabajo realizado.

Por otro lado, el rol que desempeña una persona que integra un equipo de evaluación externa es preponderante, pues debe ser firme y segura de su posición al emitir opiniones y específicamente reconocer la delicada posición en que se está desempeñando, ya que en ese momento, representa un rol de poder que le sitúa en una posición estratégica, desde la que puede legitimar o por el contrario, menoscabar el proceso de autoevaluación que se está realizando. 


\section{Referencias}

Centro de Evaluación Académica. (2004). Documento orientador para el asesoramiento de los procesos de autoevaluación-autorregulación y la acreditación. Lineamientos conceptuales y procedimientos generales sobre Autoevaluación-Autorregulación en la Universidad de Costa Rica. San José: Vicerrectoría de Docencia. Universidad de Costa Rica.

Centro de Evaluación Académica. (1999) El proceso de autoevaluación para la acreditación. Una guía orientadora. San José: Vicerrectoría de Docencia. Universidad de Costa Rica.

Consejo Superior Universitario Centroamericano (CSUCA). (2000). Guía para la validación externa del proceso de autoevaluación. San José, Costa Rica: Sistema Centroamericano de Evaluación y Acreditación de la Educación Superior (SICEVAES). Comisión Técnica de Evaluación.

Consejo Superior Universitario Centroamericano (CSUCA). (2001). Guía de evaluación de la calidad de los programas para el equipo de evaluación externa. Panamá: SICAR/CSUCA. Programas Regionales Centroamericanos. Comisión Técnica de Evaluación del Sistema de Carreras Regionales del CSUCA.

Comisión Nacional de Acreditación de Pregrado. (2001). Manual para el desarrollo de procesos de autoevaluación. Chile: Ministerio de Educación.

González G., Jorge et al. (2000). Análisis estructural integrativo de organizaciones universitarias. México: Comité de Ciencias Naturales y Exactas de los Comités Interinstitucionales para la Evaluación de la Educación Superior.

Glazman Nowalski, Raquel. (2001). Evaluación y exclusión en la enseñanza universitaria. México D.F.: Paidós Educador.

Grad Fuchel, Héctor. (1998). Experiencia de evaluación externa de las universidades. En: Revista de Educación. No. 316. Madrid, España. pp. 319-328.

Kells R., Herbert. (1997) Procesos de autoevaluación en la educación superior. Perú: Fondo Editorial. Pontificia Universidad Católica del Perú.

Peralta, Teresita. (s.f.). Guía para la evaluación externa por pares académicos. San José: Comisión Técnica de Evaluación (CSUCA).

Revista de Ciencias Sociales. (2001) Evaluación y acreditación en la enseñanza superior. Números 92-93. Universidad de Costa Rica.

Santos, Miguel A. (1993) Hacer visible lo cotidiano. Teoría práctica de la evaluación cualitativa de centros escolares. Madrid, España: Ediciones Akal. 
Silva, Moisés et al. (1999). Principios y elementos operativos de autoevaluación en la educación universitaria. Santiago de Chile: Publicación de la Oficina de Autoevaluación Institucional. Universidad de Concepción. Chile.

SINAES. (2000). Informe de pares externos. San José, Costa Rica: Proceso de Evaluación Externa, S.D.

Sistema Nacional de Acreditación de la Educación Superior. SINAES. (2000). Términos de referencia para la contratación de pares académicos. San José, Costa Rica: SINAES.

UDUAL. (2000). 1er Taller Latinoamericano de Evaluadores Externos. CIEES. México: Universidad Autónoma del Estado de Morelos.

UDUAL. (2000). 2do Taller Latinoamericano de Evaluadores Externos. CIEES. México: Universidad de Guanajuato. Estado de Guanajuato.

Vicerrectoría de Docencia. (2001). Resolución No. 7042-2001. San José: Universidad de Costa Rica. 